PROCEEDINGS OF THE

AMERICAN MATHEMATICAL SOCIETY

Volume 134, Number 2, Pages 475-485

S 0002-9939(05)08130-X

Article electronically published on July 7, 2005

\title{
REPRESENTATION OF CONTRACTIVELY COMPLEMENTED HILBERTIAN OPERATOR SPACES ON THE FOCK SPACE
}

\author{
MATTHEW NEAL AND BERNARD RUSSO
}

(Communicated by David R. Larson)

\begin{abstract}
The operator spaces $H_{n}^{k}, 1 \leq k \leq n$, generalizing the row and column Hilbert spaces, and arising in the authors' previous study of contractively complemented subspaces of $C^{*}$-algebras, are shown to be homogeneous and completely isometric to a space of creation operators on a subspace of the anti-symmetric Fock space. The completely bounded Banach-Mazur distance from $H_{n}^{k}$ to a row or column space is explicitly calculated.
\end{abstract}

\section{INTRODUCTION AND PRELIMINARIES}

A well-known result of Friedman and Russo ([4, Theorem 2]) states that if a subspace $X$ of a $C^{*}$-algebra $A$ is the range of a contractive projection on $A$, then $X$ is isometric to a $J C^{*}$-triple, that is, a norm closed subspace of $B(H, K)$ stable under the triple product $a b^{*} c+c b^{*} a$. If $X$ is atomic (in particular, finite dimensional), then it is isometric to a direct sum of Cartan factors of types 1 to 4 .

The authors showed in [7] that this latter result fails, as it stands, in the category of operator spaces. In that paper, we defined a family of $n$-dimensional Hilbertian operator spaces $H_{n}^{k}, 1 \leq k \leq n$, generalizing the row and column Hilbert spaces $R_{n}$ and $C_{n}$ and showed that in the above result, if $X$ is atomic, the word "isometric" can be replaced by "completely semi-isometric," provided the spaces $H_{n}^{k}$ are allowed as summands along with the Cartan factors ([7, Theorem 2]). It is pointed out in [7] that the space $H_{n}^{k}$ is contractively complemented in some $B(K)$, and for $1<k<n$, is not completely (semi-)isometric to either of the Cartan factors $B\left(\mathbf{C}, \mathbf{C}^{n}\right)=H_{n}^{1}$ or $B\left(\mathbf{C}^{n}, \mathbf{C}\right)=H_{n}^{n}$, and that these spaces appeared in a slightly different form and context in [1]. It is also shown in [7, Theorem 3] that finite-dimensional $J C^{*}$ triples which are contractively complemented in a $C^{*}$-algebra can be classified up to complete isometry.

In this paper, we study the operator space structure of the spaces $H_{n}^{k}$. Besides being a generalization of the row and column Hilbert spaces, as shown in Lemma 2.1 below, they are completely isometric to the span of creation operators on a subspace of the anti-symmetric Fock space. Thus they are related to the operator

Received by the editors September 22, 2004.

2000 Mathematics Subject Classification. Primary 46L07.

Key words and phrases. Hilbertian operator space, homogeneous operator space, contractive projection, creation operator, anti-symmetric Fock space, completely bounded Banach-Mazur distance.

(C)2005 American Mathematical Society Reverts to public domain 28 years from publication 
space denoted by $\Phi_{n}$ in [9, section 9.3], which is the span of the creation operators on the full anti-symmetric Fock space. $\Phi_{n}$ is the unique operator space which is completely isometric to the span of $n$ operators satisfying the canonical anticommutation relations (CAR), [9, Theorem 9.3.1], and $\bigcap_{k=1}^{n} H_{n}^{k}$ is completely isometric to $\Phi_{n}$. We show in Theorem 2 below that all finite-dimensional Hilbertian operator spaces $X$ which are contractively complemented in some $C^{*}$-algebra are completely isometric to the diagonal of two spaces, one space being an intersection of some of the spaces $H_{n}^{k}$ and the other space lying in the kernel of the projection which maps onto $X$. Since any intersection of the spaces $H_{n}^{k}$ is also completely isometric to a space of creation operators on a subspace of the full anti-symmetric Fock space, Theorem 2 can be interpreted as saying that every contractively complemented Hilbertian operator space is, up to complete isometry, essentially a space of creation operators. This result is analogous to the result of Robertson, 10, which states that every completely contractively complemented Hilbertian operator space is completely isometric to either row or column space.

The operator space structures of the row and column Hilbert spaces $R_{n}$ and $C_{n}$ have been well studied, and in particular it is known that they are homogeneous, dual to each other in the operator space sense, and have completely bounded Banach-Mazur distance $n$ between them. We show here that $H_{n}^{k}$ is homogeneous (Theorem 11), and we give an explicit formula for the completely bounded BanachMazur distance from it to $R_{n}=H_{n}^{n}$ and $C_{n}=H_{n}^{1}$ (Theorem 3). This answers a question we posed in [7] and shows, interestingly, that the points $R_{n}, C_{n}$ and $H_{n}^{k}$ lie on a straight line in the metric space of all operator spaces of dimension $n$.

Recall that a Cartan factor of type 1 is $B(H, K)$ for complex Hilbert spaces $H$ and $K$. To define the Cartan factors of types 2 and 3 , fix a conjugation $J$ on a complex Hilbert space $H$, that is, a conjugate-linear isometry of order 2, and for $x \in B(H)$, let $x^{t}=J x^{*} J$. A Cartan factor of type 2 (respectively of type 3 ) is $A(H, J)=\left\{x \in B(H): x^{t}=-x\right\}$ (respectively $S(H, J)=\left\{x \in B(H): x^{t}=-x\right\}$ ). A Cartan factor of type 4 is the spin factor (cf. [7, Subsection 3.1]).

An operator space is a subspace $X$ of $B(H)$, the space of bounded linear operators on a complex Hilbert space. Its operator space structure is given by the sequence of norms on the set of matrices $M_{n}(X)$ with entries from $X$, determined by the identification $M_{n}(X) \subset M_{n}(B(H))=B(H \oplus H \oplus \cdots \oplus H)$. See [9] for the general theory of operator spaces, which is now extensive and covered in several other monographs, for example [3], 8], and [2]. Let us just recall that a linear mapping $\varphi: X \rightarrow Y$ between two operator spaces is completely bounded if the induced mappings $\varphi_{n}: M_{n}(X) \rightarrow M_{n}(Y)$ defined by $\varphi_{n}\left(\left[x_{i j}\right]\right)=\left[\varphi\left(x_{i j}\right)\right]$ satisfy $\|\varphi\|_{\mathrm{cb}}:=$ $\sup _{n}\left\|\varphi_{n}\right\|<\infty$. A completely bounded map is a completely bounded isomorphism if its inverse exists and is completely bounded. Two operator spaces are completely isometric if there is a linear isomorphism $T$ between them with $\|T\|_{\mathrm{cb}}=\left\|T^{-1}\right\|_{\mathrm{cb}}=$ 1 . We call $T$ a complete isometry in this case.

In the matrix representation for $B\left(\ell^{2}\right)$ consider the column Hilbert space $C=$ $\overline{\mathrm{sp}}\left\{e_{i 1}: i \geq 1\right\}$ and the row Hilbert space $R=\overline{\mathrm{sp}}\left\{e_{1 j}: j \geq 1\right\}$ and their finitedimensional versions $C_{n}=\operatorname{sp}\left\{e_{i 1}: 1 \leq i \leq n\right\}$ and $R_{n}=\operatorname{sp}\left\{e_{1 j}: 1 \leq j \leq n\right\}$. Here of course $e_{i j}$ is the operator defined by the matrix with a 1 in the $(i, j)$ entry and zeros elsewhere. Although $R$ and $C$ are Banach isometric, they are not completely isomorphic; and $R_{n}$ and $C_{n}$, while completely isomorphic, are not completely isometric. 
An operator space is said to be homogeneous if every bounded linear map on it is completely bounded with the norm and completely bounded norm coinciding (see [9, 9.2]), and it is Hilbertian if it is isometric to a Hilbert space. A linear map of one operator space into another is said to be a complete semi-isometry if it is isometric and completely contractive. The completely bounded Banach-Mazur distance between two (completely isomorphic) operator spaces $E, F$ is defined by

$$
\mathrm{d}_{\mathrm{cb}}(E, F)=\inf \left\{\|u\|_{\mathrm{cb}}\left\|u^{-1}\right\|_{\mathrm{cb}}: u: E \rightarrow F \text { complete isomorphism }\right\} .
$$

This paper is organized as follows. In section 1, we show that the spaces $H_{n}^{k}$ are homogeneous operator spaces. Although we use some multilinear algebra, our proof is direct and does not make use of the identification of $H_{n}^{k}$ with a space of creation operators. In section 2 we establish the complete isometry of $H_{n}^{k}$ with a space of creation operators and use it to describe the fine structure of the range of a contractive projection on a $C^{*}$-algebra in case said range is isometric to a Hilbert space. We also establish some spectral properties of creation operators. In section 3 we explicitly compute the completely bounded Banach-Mazur distance from the space $H_{n}^{k}$ to the column and row Hilbert spaces $H_{n}^{1}$ and $H_{n}^{n}$ and state some problems for further study.

\section{Homogeneity OF THE SPACES $H_{n}^{k}$}

We begin by recalling from [7, Sections 6,7] the construction of the spaces $H_{n}^{k}$, $1 \leq k \leq n$. Let $I$ denote a subset of $\{1,2, \ldots, n\}$ of cardinality $|I|=k-1$. The number of such $I$ is $q:=\left(\begin{array}{c}n \\ k-1\end{array}\right)$. Let $J$ denote a subset of $\{1,2, \ldots, n\}$ of cardinality $|J|=n-k$. The number of such $J$ is $p:=\left(\begin{array}{c}n \\ n-k\end{array}\right)$. Unless otherwise noted, we shall assume that each $I=\left\{i_{1}, \ldots, i_{k-1}\right\}$ is such that $i_{1}<\cdots<i_{k-1}$, and that the collection $\left\{I_{1}, \ldots, I_{q}\right\}$ of all such subsets is ordered lexicographically. Similarly, if $J=\left\{j_{1}, \ldots, j_{n-k}\right\}$, then $j_{1}<\cdots<j_{n-k}$ and $\left\{J_{1}, \ldots, J_{p}\right\}$ is ordered lexicographically.

We shall use the notation $e_{i}$ to denote the column vector with a 1 in the $i^{\text {th }}$ position and zeros elsewhere. Thus $e_{1}, \ldots, e_{n}$ denotes the canonical basis of column vectors for $\mathbf{C}^{n}$, and for example $e_{J_{1}}, \ldots, e_{J_{p}}$ denotes the canonical basis of column vectors for $\mathbf{C}^{p}$.

The space $H_{n}^{k}$ is the linear span of matrices $b_{i}^{n, k}, 1 \leq i \leq n$, given by

$$
b_{i}^{n, k}=\sum_{I \cap J=\emptyset,(I \cup J)^{c}=\{i\}} \epsilon(I, i, J) e_{J, I},
$$

where $e_{J, I}=e_{J} \otimes e_{I}=e_{J} e_{I}^{t} \in M_{p, q}(\mathbf{C})=B\left(\mathbf{C}^{q}, \mathbf{C}^{p}\right)$, and $\epsilon(I, i, J)$ is the signature of the permutation taking $\left(i_{1}, \ldots, i_{k-1}, i, j_{1}, \ldots, j_{n-k}\right)$ to $\left.(1, \ldots, n) 1\right]$ Since the $b_{i}^{n, k}$ are the image under a triple isomorphism (actually ternary isomorphism) of a rectangular grid in a $J W^{*}$-triple of rank one, they form an orthonormal basis for $H_{n}^{k}$ (cf. the beginning of subsection 5.3 and the beginning of section 7 of [7]).

In the rest of this section, we shall use the following lemma about determinants, whose proof can be found, for example, in [1].

Lemma 1.1. Let $X=\left[\xi_{i j}\right]$ be an $n \times m$ matrix. Let $H \subset\{1, \ldots, n\}$ and $K \subset$ $\{1, \ldots, m\}$ both have cardinality $r \leq \min \{n, m\}$. Let $X_{H, K}$ denote the corresponding $r \times r$ submatrix.

\footnotetext{
${ }^{1}$ In [7], $\epsilon(I, i, J)$ is also denoted by $\epsilon(I, J)$. However, in this paper, $\epsilon(I, J)$ will denote the signature of the permutation taking $\left(i_{1}, \ldots, i_{k-1}, j_{1}, \ldots, j_{n-k}\right)$ to $(1, \ldots, \hat{i}, \ldots, n)$.
} 
(i) If $x_{i}=\sum_{j=1}^{n} \xi_{j i} e_{j} \in \mathbf{C}^{n}$ for $1 \leq p \leq n$, then $x_{1} \wedge \cdots \wedge x_{p}=\sum_{H} \operatorname{det} X_{H, L} e_{H}$, where the sum is over all $H$ of cardinality $p, L=\{1, \ldots, p\}$, and $X$ is the $n \times p$ matrix $\left[\xi_{i j}\right]$. (Prop. 3.3, page 84 of [11.)

(ii) If $X$ is an $n \times n$ matrix and $H \subset\{1, \ldots, n\}$, let $H^{\prime}$ denote the complement of $H$. Then $\operatorname{det} X=\epsilon\left(H, H^{\prime}\right) \sum_{R} \epsilon\left(R, R^{\prime}\right) \operatorname{det} X_{R, H} \operatorname{det} X_{R^{\prime}, H^{\prime}}$, where the sum is over all sets $R$ having the same cardinality of $H$. (Prop. 3.4(1) page 87 of [11.)

(iii) If $H, K \subset\{1, \ldots, n\}$ have cardinality $r$ and $n-r$, and $H \cap K \neq \emptyset$, then $\sum_{R} \epsilon\left(R, R^{\prime}\right) \operatorname{det} X_{R, H} \operatorname{det} X_{R^{\prime}, K}=0$, where the sum is over all sets $R$ having cardinality $r$. (Prop. 3.4(2), page 87 of [11].)

Let $e_{1}, \ldots, e_{n}$ be the canonical basis for the column Hilbert space $C_{n}=M_{n, 1}(\mathbf{C})$ $=B\left(\mathbf{C}, \mathbf{C}^{n}\right)$ and define an isometry $\psi: C_{n} \rightarrow H_{n}^{k}$ via $\psi\left(e_{i}\right)=b_{i}^{n, k}, 1 \leq i \leq n$. Let $u=\left[\begin{array}{lll}u_{1} & \cdots & u_{n}\end{array}\right]$ be a unitary matrix so that $u_{1}, \ldots, u_{n}$ is an orthonormal basis for $C_{n}$. Then, with $u_{i}=\sum_{j=1}^{n} u_{j i} e_{j}$, we have

$$
u=\left[\begin{array}{lll}
u_{11} & \cdots & u_{1 n} \\
\vdots & \cdots & \vdots \\
u_{n 1} & \cdots & u_{n n}
\end{array}\right]
$$

and

$$
\psi\left(u_{i}\right)=\sum_{j=1}^{n} u_{j i} b_{j}^{n, k}=\sum_{j=1}^{n} u_{j i} \sum_{I \cap J=\emptyset,(I \cup J)^{c}=\{i\}} \epsilon(I, i, J) e_{J, I} .
$$

Lemma 1.2. The $\left(J^{\prime}, I^{\prime}\right)$-entry of the $p \times q$ matrix $\psi\left(u_{i}\right)$ is given by

$$
\left(\psi\left(u_{i}\right)\right)_{J^{\prime}, I^{\prime}}=\sum_{I \cap J=\emptyset,(I \cup J)^{c}=\{i\}} \epsilon(I, i, J) \frac{\operatorname{det} \bar{u}_{J^{\prime}, J} \operatorname{det} \bar{u}_{I^{\prime}, I}}{\operatorname{det} \bar{u}},
$$

where $\bar{u}$ is the complex conjugate of $u$.

Proof. Let us first calculate the left side of (10):

$$
\begin{aligned}
\left(\psi\left(u_{i}\right)\right)_{J^{\prime}, I^{\prime}} & =e_{J^{\prime}}^{t} \psi\left(u_{i}\right) e_{I^{\prime}} \\
& =\sum_{j=1}^{n} u_{j i} \sum_{I \cap J=\emptyset,(I \cup J)^{c}=\{j\}} \epsilon(I, j, J) e_{J^{\prime}}^{t} e_{J} e_{I}^{t} e_{I^{\prime}} \\
& = \begin{cases}0, & J^{\prime} \cap I^{\prime} \neq \emptyset, \\
u_{l i} \epsilon\left(I^{\prime}, l, J^{\prime}\right), & J^{\prime} \cap I^{\prime}=\emptyset,\left(I^{\prime} \cup J^{\prime}\right)^{c}=\{l\} .\end{cases}
\end{aligned}
$$

Before calculating the right side of (1), note that $\epsilon(I, i, J) \epsilon(I, J)=(-1)^{i+k}$; indeed,

$$
\begin{aligned}
\epsilon(I, i, J) \epsilon(I, J) & =(-1)^{k-1} \epsilon(i, I, J) \epsilon(I, J) \\
& =(-1)^{k-1} \epsilon(I, J) \epsilon(i, 1,2, \ldots, \hat{i}, \ldots, n-1) \epsilon(I, J) \\
& =(-1)^{k-1}(-1)^{i-1}=(-1)^{k+i} .
\end{aligned}
$$

Therefore, the right side of (1) is equal to

$$
\begin{aligned}
& \sum_{I \cap J=\emptyset,(I \cup J)^{c}=\{i\}} \epsilon(I, i, J) \epsilon\left(I^{\prime}, J^{\prime}\right) \epsilon\left(I^{\prime}, J^{\prime}\right) \epsilon(I, J) \epsilon(I, J) \frac{\operatorname{det} \bar{u}_{J^{\prime}, J} \operatorname{det} \bar{u}_{I^{\prime}, I}}{\operatorname{det} \bar{u}} \\
= & (-1)^{k+i} \epsilon\left(I^{\prime}, J^{\prime}\right) \sum_{I \cap J=\emptyset,(I \cup J)^{c}=\{i\}} \epsilon\left(I^{\prime}, J^{\prime}\right) \epsilon(I, J) \frac{\operatorname{det} \bar{u}_{J^{\prime}, J} \operatorname{det} \bar{u}_{I^{\prime}, I} .}{\operatorname{det} \bar{u}}
\end{aligned}
$$


According to Lemma 1.1(iii), the above sum is 0 if $J^{\prime} \cap I^{\prime} \neq \emptyset$. Otherwise, if $J^{\prime} \cap I^{\prime}=\emptyset$ so that $\left(I^{\prime} \cup J^{\prime}\right)^{c}=\{l\}$, the right side of (1) equals

$$
\frac{(-1)^{k-l} \epsilon\left(I^{\prime}, J^{\prime}\right)(-1)^{i+l}}{\operatorname{det} \bar{u}} \sum_{I \cap J=\emptyset,(I \cup J)^{c}=\{i\}} \epsilon\left(I^{\prime}, J^{\prime}\right) \epsilon(I, J) \operatorname{det} \bar{u}_{J^{\prime}, J} \operatorname{det} \bar{u}_{I^{\prime}, I} .
$$

Now by Lemma 1.1(ii), the above sum is the determinant of the $(l, i)$-minor of the matrix $\bar{u}$, call this $\bar{M}_{l i}$. Thus, for $J^{\prime} \cap I^{\prime}=\emptyset$, the right side of (1) is equal to

$$
\begin{aligned}
\epsilon\left(I^{\prime}, l, J^{\prime}\right)\left[\frac{(-1)^{i+l} \operatorname{det} \bar{M}_{l i}}{\operatorname{det} \bar{u}}\right] & =\epsilon\left(I^{\prime}, l, J^{\prime}\right) \times[\text { the }(i, l) \text {-entry of the inverse of } \bar{u}] \\
& =\epsilon\left(I^{\prime}, l, J^{\prime}\right) u_{l i} .
\end{aligned}
$$

Theorem 1. $H_{n}^{k}$ is a homogeneous operator space.

Proof. Let $\alpha$ be a unitary operator on $H_{n}^{k}$. To prove the theorem, it suffices, by [9, Prop. 9.2.1], to show that $\alpha$ is a complete isometry. We shall show that $\alpha(x)=\lambda v x w$ for suitable unitary matrices $v$ and $w$, and $\lambda \in \mathbf{C}$, with $|\lambda|=1$, which will complete the proof.

Recall that $\psi: C_{n} \rightarrow H_{n}^{k}$ is the isometry defined by $\psi\left(e_{i}\right)=b_{i}^{n, k}$. Let $\psi^{-1} \alpha \psi$ have matrix $u^{-1}$ on $C_{n}$ with respect to the basis $e_{1}, \ldots, e_{n}$. As in Lemma 1.2, let $u_{1}, \ldots, u_{n}$ be the columns of $u$. We shall show that $\alpha(x)=\lambda v x w$ holds for every $x \in H_{n}^{k}$, where $\lambda=\operatorname{det} \bar{u}, w=\left[\begin{array}{lll}\wedge_{i \in I_{1}} u_{i} & \cdots & \wedge_{i \in I_{q}} u_{i}\end{array}\right]$, and

$$
v=\left[\begin{array}{c}
\left(\wedge_{j \in J_{1}} u_{j}\right)^{t} \\
\vdots \\
\left(\wedge_{j \in J_{p}} u_{j}\right)^{t}
\end{array}\right]
$$

(The fact that $v$ and $w$ are unitary matrices follows from the definition of the inner product on $\wedge^{r} \mathbf{C}^{n}:\left(x_{1} \wedge \cdots \wedge x_{r} \mid y_{1} \wedge \cdots \wedge y_{r}\right)=\operatorname{det}\left[\left(x_{i} \mid y_{j}\right)\right]$.)

In the first place, $\psi^{-1} \alpha \psi\left(u_{i}\right)=u^{-1}\left(u_{i}\right)=e_{i}$, so that $\alpha \psi\left(u_{i}\right)=\psi\left(e_{i}\right)=b_{i}^{n, k}$. Thus it suffices to prove

$$
v \psi\left(u_{i}\right) w=b_{i}^{n, k} / \operatorname{det} \bar{u}
$$

Let us first show that

$$
\psi\left(u_{i}\right)=(\operatorname{det} \bar{u})^{-1} \sum_{I \cap J=\emptyset,(I \cup J)^{c}=\{i\}} \epsilon(I, i, J)\left(\wedge_{j \in J} \bar{u}_{j}\right)\left(\wedge_{i \in I} \bar{u}_{i}\right)^{t} .
$$

By Lemma 1.2, the proof of (3) amounts to

$$
\left[\left(\wedge_{j \in J} u_{j}\right)\left(\wedge_{i \in I} u_{i}\right)^{t}\right]_{J^{\prime}, I^{\prime}}=\operatorname{det} u_{J^{\prime}, J} \operatorname{det} u_{I^{\prime}, I} .
$$

The left side of (4) is given by $e_{J^{\prime}}^{t}\left(\wedge_{j \in J} u_{j}\right)\left(\wedge_{i \in I} u_{i}\right)^{t} e_{I^{\prime}}$. By Lemma 1.1(i),

$$
\wedge_{j \in J} u_{j}=\sum_{L} \operatorname{det} u_{L, J} e_{L}
$$

where $L$ runs over the subsets of cardinality $n-k$. Hence

$$
e_{J^{\prime}}^{t}\left(\wedge_{j \in J} u_{j}\right)=\sum_{L} \operatorname{det} u_{L, J} e_{J^{\prime}}^{t} e_{L}=\operatorname{det} u_{J^{\prime}, J}
$$

Similarly, $\left(\wedge_{i \in I} u_{i}\right)^{t} e_{I^{\prime}}=\operatorname{det} u_{I^{\prime}, I}$, which proves (44) and hence (33). 
We now use (3) to prove (2). Note that since $e_{J, I}=e_{J} \otimes \bar{e}_{I}=e_{J}\left(e_{I}\right)^{t}=$ $\left(\wedge_{j \in J} e_{j}\right)\left(\wedge_{i \in I} e_{i}\right)^{t}$, we may write

$$
b_{i}^{n, k}=\sum_{I \cap J=\emptyset,(I \cup J)^{c}=\{i\}} \epsilon(I, i, J)\left(\wedge_{j \in J} e_{j}\right)\left(\wedge_{i \in I} e_{i}\right)^{t} .
$$

By (3) and (5), it suffices to prove

$$
v\left(\wedge_{j \in J} \bar{u}_{j}\right)\left(\wedge_{i \in I} \bar{u}_{i}\right)^{t} w=\left(\wedge_{j \in J} e_{j}\right)\left(\wedge_{i \in I} e_{i}\right)^{t} .
$$

This is a simple calculation. Suppose for definiteness that $J=J_{r}$ and $I=I_{s}$. Then $v\left(\wedge_{j \in J} \bar{u}_{j}\right)=e_{J_{r}}$, and $\left(\wedge_{i \in I} \bar{u}_{i}\right)^{t} w=e_{I_{s}}^{t}$.

Remark 1.3. In [7, page 2230], we defined an operator space construction denoted by $\operatorname{Diag}\left(H_{n}^{k_{1}}, \ldots, H_{n}^{k_{m}}\right)$ which depended on a choice of orthonormal basis for each of the spaces $H_{n}^{k_{j}}$. Because of the homogeneity of the spaces $H_{n}^{k}$ proved in Theorem 1 , this space is independent of these choices up to complete isometry and is now seen to be the intersection $H_{n}^{k_{1}} \cap \cdots \cap H_{n}^{k_{m}}$ in the sense of operator space theory (9, page 55]).

\section{Anti-Symmetric Fock spaCes}

Let $C_{h}^{n, k}$ denote the wedge (or creation) operator from $\wedge^{k-1} \mathbf{C}^{n}$ to $\wedge^{k} \mathbf{C}^{n}$ given by

$$
C_{h}^{n, k}\left(h_{1} \wedge \cdots \wedge h_{k-1}\right)=h \wedge h_{1} \wedge \cdots \wedge h_{k-1} .
$$

Many properties of these classical operators on the full anti-symmetric Fock space are given in [5, Exercises 12.4.39-40]. See also Lemma 2.3

As in section 1, let $e_{1}, \ldots, e_{n}$ be the usual column vector orthonormal basis for $\mathbf{C}^{n}$, and let $\left\{e_{I_{1}}, \ldots, e_{I_{q}}\right\}$ and $\left\{e_{J_{1}}, \ldots, e_{J_{p}}\right\}$ be the column vector orthonormal bases for $\mathbf{C}^{q}$ and $\mathbf{C}^{p}$ respectively, and define the unitary operators $U_{j}^{n}(j=k-1$ and $j=n-k), W_{k}^{n}, V_{k}^{n}$ in the diagram below as follows:

- $U_{k-1}^{n}\left(e_{I}\right)=e_{i_{1}} \wedge \cdots \wedge e_{i_{k-1}}$, where $I=\left\{i_{1}<\cdots<i_{k-1}\right\}$.

- $U_{n-k}^{n}\left(e_{J}\right)=e_{j_{1}} \wedge \cdots \wedge e_{j_{n-k}}$, where $J=\left\{j_{1}<\cdots<j_{n-k}\right\}$.

- $V_{k}^{n}\left(e_{i_{1}} \wedge \cdots \wedge e_{i_{k}}\right)=e_{j_{1}} \wedge \cdots \wedge e_{j_{n-k}}$, where $\left\{j_{1}<\cdots<j_{n-k}\right\}$ is the complement of $\left\{i_{1}<\cdots<i_{k}\right\}$.

- $W_{k}^{n}\left(e_{j_{1}} \wedge \cdots \wedge e_{j_{n-k}}\right)=\epsilon(i, I) \epsilon(I, i, J) e_{j_{1}} \wedge \cdots \wedge e_{j_{n-k}}$ for any $i$ and $I$ such that $I \cap J=\emptyset$ and $(I \cup J)^{c}=\{i\}$ (which is independent of the choice of $i$ or $I)$.

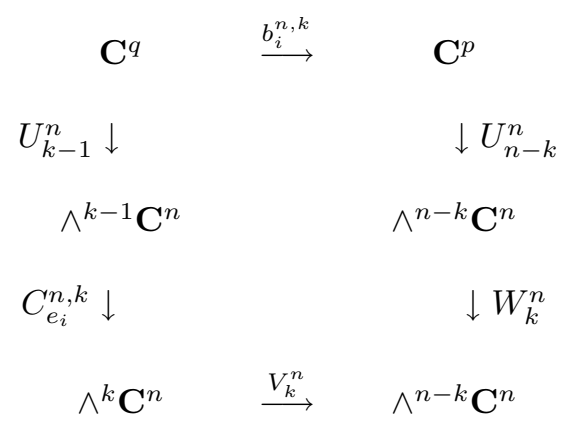

Note that since $b_{i}^{n, k}$ is a $p \times q$ matrix, it is viewed as an operator from $\mathbf{C}^{q}$ to $\mathbf{C}^{p}$. In the definition of $W_{k}^{n}, \epsilon(i, I)$ is the signature of the permutation $\left(i, i_{1}, \ldots, i_{k-1}\right) \mapsto$ 
$\left(i_{1}, \ldots, i, \ldots, i_{k-1}\right)$. To prove the non-dependence on $i$, suppose $i, i^{\prime} \notin J$. Then

$$
\begin{aligned}
\epsilon(I, i, J) & =\epsilon\left(i_{1}, \ldots, i_{k-1}, i, j_{1}, \ldots, j_{n-k}\right) \\
& =(-1)^{k-1} \epsilon\left(i, i_{1}, \ldots, i_{k-1}, j_{1}, \ldots, j_{n-k}\right) \\
& =(-1)^{k-1} \epsilon(i, I) \epsilon\left(i_{1}, \ldots, i, \ldots, i_{k-1}, j_{1}, \ldots, j_{n-k}\right),
\end{aligned}
$$

where $i_{1}<\cdots<i<\cdots<i_{k-1}$. Similarly,

$$
\epsilon\left(I^{\prime}, i^{\prime}, J\right)=(-1)^{k-1} \epsilon\left(i^{\prime}, I^{\prime}\right) \epsilon\left(i_{1}^{\prime}, \ldots, i^{\prime}, \ldots, i_{k-1}^{\prime}, j_{1}, \ldots, j_{n-k}\right),
$$

where $i_{1}^{\prime}<\cdots<i^{\prime}<\cdots<i_{k-1}^{\prime}$. Hence, $\epsilon(i, I) \epsilon(I, i, J)=\epsilon\left(i^{\prime}, I^{\prime}\right) \epsilon\left(I^{\prime}, i^{\prime}, J\right)$.

It is now a simple matter to check that $W_{k}^{n} U_{n-k}^{n} b_{i}^{n, k}=V_{k}^{n} C_{e_{i}}^{n, k} U_{k-1}^{n}$. Indeed, for any $I^{\prime}, W_{k} U_{n-k}^{n} b_{i}^{n, k}\left(e_{I^{\prime}}\right)=W_{k} U_{n-k}^{n} \epsilon\left(I^{\prime}, i, J\right)\left(e_{J}\right)=W_{k}\left(\epsilon\left(I^{\prime}, i, J\right) e_{j_{1}} \wedge \cdots \wedge\right.$ $e_{j_{n-k}}=\epsilon\left(i, I^{\prime}\right) e_{j_{1}} \wedge \cdots \wedge e_{j_{n-k}}$, and $V_{k}^{n} C_{e_{i}}^{n, k} U_{k-1}^{n}\left(e_{I^{\prime}}\right)=V_{k}^{n}\left(e_{i} \wedge e_{i_{1}^{\prime}} \wedge \cdots \wedge e_{i_{k-1}^{\prime}}\right)=$ $V_{k}^{n}\left(\epsilon\left(i, I^{\prime}\right) e_{i_{1}^{\prime}} \wedge \cdots \wedge e_{i} \wedge \cdots \wedge e_{i_{k-1}^{\prime}}\right)=\epsilon\left(i, I^{\prime}\right) e_{j_{1}} \wedge \cdots \wedge e_{j_{n-k}}$.

Hence, letting $\mathcal{C}^{n, k}$ denote the space $\operatorname{sp}\left\{C_{e_{i}}^{n, k}\right\}$ yields the following lemma.

Lemma 2.1. $H_{n}^{k}$ is completely isometric to $\mathcal{C}^{n, k}$.

By [7, Theorem 2, Corollary 2.8], every atomic contractively complemented subspace $X$ of a $C^{*}$-algebra is isometrically completely contractive to a direct sum of Cartan factors of types 1 to 4 and some of the spaces $H_{n}^{k}$. The following theorem gives more detailed information on what can be said up to complete isometry in the case of a Hilbertian $X$.

Recall that a linear map of one operator space into another is said to be a complete semi-isometry if it is isometric and completely contractive.

Theorem 2. Let $X$ be the range of a contractive projection $P$ on a $C^{*}$-algebra $A$, and suppose that $X$ is isometric to a Hilbert space. Then there exist projections $p, q \in A^{* *}$ such that

(a) $X=\{p x q+(1-p) x(1-q): x \in X\}$.

(b) The map $\mathcal{E}_{0} x=p x q$ is a complete semi-isometry of $X$ onto $p X q$.

(c) If $X$ is finite dimensional, then $p X q$ is completely isometric to an intersection of the spaces $\mathcal{C}^{n, k}$. If $X$ is infinite dimensional, then $p X q$ is completely semi-isometric to either row or column Hilbert space.

(d) Both $X$ and $p X q$ are completely isometric to the range of a contractive projection on $B(K)$ for an appropriate Hilbert space $K$.

(e) $P^{* *}(p x q)=x$ and $P^{* *}((1-p) x(1-q))=0$, for $x \in P(A)$. Hence $P^{* *}$ : $p X q \rightarrow X$ is the inverse of $\mathcal{E}_{0}$ and $(1-p) X(1-q) \subset \operatorname{ker} P^{* *}$.

Proof. (a) Since $P(A)$ is reflexive,

$$
X=P(A)=P^{* *}\left(A^{* *}\right)=p X q+(1-p) X(1-q),
$$

with the last equality following from [4, Prop. 4].

(b) By [7, Lemma 2.2].

(c) By Lemma 2.1 and [7, Prop. 2.6].

(d) For $X$ this follows directly from [7, Corollary 2.8], and for $p X q$ it follows directly from [7, Theorem 3(b) and Corollary 7.3] 2

\footnotetext{
${ }^{2}$ The authors take this opportunity to point out the following correction to [7 Lemma 7.2 and Corollary 7.3]. The term $\left(\begin{array}{l}n-1 \\ k-1\end{array}\right)^{1 / 2}$ should be replaced by $\left(\begin{array}{l}n-1 \\ k-1\end{array}\right)$ in the statements of Lemma 7.2 and Corollary 7.3, and in the proof of Lemma 7.2. Accordingly, in the proof of Corollary 7.3, $m^{1 / 2}$ should be replaced by $m$.
} 
(e) For $x \in P(A), P^{* *}(p x q) \in P^{* *}\left(A^{* *}\right)=P(A)$, say $P^{* *}(p x q)=y \in P(A)$ and by [4. Prop. 4], $y=p y q+(1-p) y(1-q)$. Thus $p\left(P^{* *}(p x q)\right) q=$ pyq. Now it follows from [4. Prop. 1], that $p\left(P^{* *} z\right) q=z$ for all $z \in$ $p P^{* *}\left(A^{* *}\right) q$. With $z=p x q$, we have $p x q=p y q$ and by (b) $x=y$, proving that $P^{* *}(p x q)=x$. Moreover, $P^{* *}((1-p) x(1-q))=P^{* *}(x-p x q)=$ $x-P^{* *}(p x q)=x-y=0$.

Remark 2.2. For any subset $S \subset\{1, \ldots, n\}, \bigcap_{k \in S} \mathcal{C}^{n, k}$ is exactly the space of creation operators on the direct sum $\bigoplus_{k \in S} \wedge^{k-1} \mathbf{C}^{n}$. So Theorem 2 says that all finite-dimensional contractively complemented Hilbertian operator spaces are essentially a space of creation operators in the anti-symmetric Fock space. In particular, if $S=\{1, \ldots, n\}$, this is the space $\Phi_{n}$ discussed in [9, section 9.3].

The following properties of the wedge operators follow easily from the definition. They will be used, together with Lemma 2.3. in section 3 .

- $C_{h}^{n, k *}\left(h_{1} \wedge \cdots \wedge h_{k}\right)=\sum_{j=1}^{k}(-1)^{j+1}\left(h_{j} \mid h\right) h_{1} \wedge \cdots \wedge h_{j-1} \wedge h_{j+1} \wedge \cdots \wedge h_{k}$.

- $C_{h}^{n, k} C_{h}^{n, k *}\left(h_{1} \wedge \cdots \wedge h_{k}\right)=\sum_{j=1}^{k}\left(h_{j} \mid h\right) h_{1} \wedge \cdots \wedge h_{j}^{\prime} \wedge \cdots \wedge h_{k} \quad\left(h_{j}^{\prime}=h\right)$, for $h \in \mathbf{C}^{n}$. In particular, $C_{h}^{n, 1} C_{h}^{n, 1 *}=h \otimes \bar{h}$.

- $C_{h}^{n, k *} C_{h}^{n, k}=\|h\|^{2} I-C_{h}^{n, k-1} C_{h}^{n, k-1 *}$, for $h \in \mathbf{C}^{n}$.

Lemma 2.3. (a) $\operatorname{tr}\left(C_{h}^{n, k *} C_{h}^{n, k}\right)=\left(\begin{array}{c}n-1 \\ k-1\end{array}\right)\|h\|^{2}$. In particular, $C_{h}^{n, 1 *} C_{h}^{n, 1}=$ $\|h\|^{2}$.

(b) The eigenvalues of $\sum_{i=1}^{m} C_{h_{i}}^{n, k} C_{h_{i}}^{n, k *}$ are precisely the sums of $k$ eigenvalues of $\sum_{i=1}^{m} C_{h_{i}}^{n, 1} C_{h_{i}}^{n, 1 *}$.

(c) The eigenvalues of $\sum_{i=1}^{m} C_{h_{i}}^{n, k *} C_{h_{i}}^{n, k}$ are precisely the sums of $n-k+1$ eigenvalues of $\sum_{i=1}^{m} C_{h_{i}}^{n, n *} C_{h_{i}}^{n, n}$.

Proof. In the first place, we have

$$
\begin{aligned}
\operatorname{tr}\left(C_{h}^{n, k} C_{h}^{n, k *}\right) & =\operatorname{tr}\left(C_{h}^{n, k *} C_{h}^{n, k}\right) \\
& =\sum_{i_{1}, \ldots, i_{k-1}}\left(C_{h}^{n, k *} C_{h}^{n, k}\left(e_{i_{1}} \wedge \cdots \wedge e_{i_{k-1}}\right) \mid e_{i_{1}} \wedge \cdots \wedge e_{i_{k-1}}\right) \\
& =\sum_{i_{1}, \ldots, i_{k-1}}\left(h \wedge e_{i_{1}} \wedge \cdots \wedge e_{i_{k-1}} \mid h \wedge e_{i_{1}} \wedge \cdots \wedge e_{i_{k-1}}\right) \\
& =\sum_{i_{1}, \ldots, i_{k-1}} \operatorname{det}\left[\begin{array}{cccc}
(h \mid h) & \left(h \mid e_{i_{1}}\right) & \cdots & \left(h \mid e_{i_{k-1}}\right) \\
\left(e_{i_{1}} \mid h\right) & 1 & \cdots & 0 \\
\cdots & \cdots & \cdots & \cdots \\
\left(e_{i_{k-1}} \mid h\right) & 0 & \cdots & 1
\end{array}\right] \\
& =\sum_{i_{1}, \ldots, i_{k-1}}\left(\|h\|^{2}-\sum_{j=1}^{k-1}\left|\left(h \mid e_{i_{j}}\right)\right|^{2}\right. \\
& =\left(\begin{array}{c}
n \\
k-1
\end{array}\right)\|h\|^{2}-\sum_{i_{1}, \ldots, i_{k-1}} \sum_{j=1}^{k-1}\left|\left(h \mid e_{i_{j}}\right)\right|^{2} \\
& =\left(\begin{array}{c}
n \\
k-1
\end{array}\right)\|h\|^{2}-\sum_{l=1}^{n}\left(\begin{array}{l}
n-1 \\
k-2
\end{array}\right)\left|\left(h \mid e_{l}\right)\right|^{2}
\end{aligned}
$$




$$
=\left(\left(\begin{array}{c}
n \\
k-1
\end{array}\right)-\left(\begin{array}{l}
n-1 \\
k-2
\end{array}\right)\right)\|h\|^{2}=\left(\begin{array}{l}
n-1 \\
k-1
\end{array}\right)\|h\|^{2} .
$$

To prove the second statement, let the (repeated) eigenvalues of $\sum_{i=1}^{m} C_{h_{i}}^{n, 1} C_{h_{i}}^{n, 1 *}$ be $\lambda_{1} \geq \cdots \geq \lambda_{n}$ and let $\xi_{1}, \ldots, \xi_{n}$ be an orthonormal basis of $\mathbf{C}^{n}$ consisting of eigenvectors of $\sum_{i=1}^{m} C_{h_{i}}^{n, 1} C_{h_{i}}^{n, 1 *}$ so that $\sum_{i}\left(\xi_{k} \mid h_{i}\right) h_{i}=\lambda_{k} \xi_{k}$. Then

$$
\begin{aligned}
\sum_{i=1}^{m} C_{h_{i}}^{n, k} C_{h_{i}}^{n, k *}\left(\xi_{i_{1}} \wedge \cdots \wedge \xi_{i_{k}}\right) & =\sum_{i} \sum_{j=1}^{k}\left(\xi_{i_{j}} \mid h_{i}\right) \xi_{i_{1}} \wedge \cdots \wedge h_{i} \wedge \cdots \wedge \xi_{i_{k}} \\
& =\sum_{j} \xi_{i_{1}} \wedge \cdots \wedge\left[\sum_{i}\left(\xi_{i_{j}} \mid h_{i}\right) h_{i}\right] \wedge \cdots \xi_{i_{k}} \\
& =\left[\sum_{j} \lambda_{i_{j}}\right] \xi_{i_{1}} \wedge \cdots \wedge \xi_{i_{k}} .
\end{aligned}
$$

Conversely, if $\xi=\sum \alpha_{i_{1}, \ldots, i_{k}} \xi_{i_{1}} \wedge \cdots \wedge \xi_{i_{k}}$ is an eigenvector of $\sum_{i=1}^{m} C_{h_{i}}^{n, k} C_{h_{i}}^{n, k *}$, with eigenvalue $\lambda$, then

$$
\begin{aligned}
\lambda & \sum \alpha_{i_{1}, \ldots, i_{k}} \xi_{i_{1}} \wedge \cdots \wedge \xi_{i_{k}} \\
& =\sum_{i=1}^{m} C_{h_{i}}^{n, k} C_{h_{i}}^{n, k *} \sum \alpha_{i_{1}, \ldots, i_{k}} \xi_{i_{1}} \wedge \cdots \wedge \xi_{i_{k}} \\
& =\sum \alpha_{i_{1}, \ldots, i_{k}} \sum_{i} \sum_{j}\left(\xi_{i_{j}} \mid h_{i}\right) \xi_{i_{1}} \wedge \cdots \wedge h_{i} \wedge \cdots \wedge \xi_{i_{k}} \\
& =\sum \alpha_{i_{1}, \ldots, i_{k}} \sum_{j} \xi_{i_{1}} \wedge \cdots \wedge\left[\sum_{i=1}^{m} C_{h_{i}}^{n, 1} C_{h_{i}}^{n, 1 *} \xi_{i_{j}}\right] \wedge \cdots \wedge \xi_{i_{k}} \\
& =\sum \alpha_{i_{1}, \ldots, i_{k}}\left[\sum_{j} \lambda_{i_{j}}\right] \xi_{i_{1}} \wedge \cdots \wedge \xi_{i_{k}} .
\end{aligned}
$$

From this, the second statement follows.

Since $C_{h}^{n, 1 *}=C_{h}^{n, n}$ and $\|h\|^{2}=\operatorname{tr}\left(C_{h}^{n, 1} C_{h}^{n, 1 *}\right)$, (c) follows from the third bullet above and (b).

\section{Completely bounded Banach-Mazur distance}

Recall that the completely bounded Banach-Mazur distance between two (completely isomorphic) operator spaces $E, F$ is defined by

$$
\mathrm{d}_{\mathrm{cb}}(E, F)=\inf \left\{\|u\|_{\mathrm{cb}}\left\|u^{-1}\right\|_{\mathrm{cb}}: u: E \rightarrow F \text { complete isomorphism }\right\} .
$$

We shall explicitly compute $\mathrm{d}_{\mathrm{cb}}\left(H_{n}^{k}, H_{n}^{1}\right)$ and $\mathrm{d}_{\mathrm{cb}}\left(H_{n}^{k}, H_{n}^{n}\right)$. By Lemma 2.1. we can identify $H_{n}^{k}$ with $\mathcal{C}^{n, k}$. For a fixed $k$, let $\psi: H_{n}^{1} \rightarrow H_{n}^{k}$ be the isometry given by $\psi\left(C_{e_{i}}^{n, 1}\right)=C_{e_{i}}^{n, k}$. Recall from [6, Prop. 4] that, since $\psi$ is a mapping from the column Hilbert space $H_{n}^{1},\|\psi\|_{\mathrm{cb}}=\|\psi\|_{\text {row-cb }}$, where $\|\psi\|_{\text {row-cb }}:=$ $\sup \left\{\left\|\left(\psi\left(h_{1}\right), \ldots, \psi\left(h_{m}\right)\right)\right\|\right\}$, where the supremum is extended over all $m \geq 1$ and all row vectors with $\left\|\left(h_{1}, \ldots, h_{m}\right)\right\| \leq 1$. Similarly, by [6, Prop. 2], $\left\|\psi^{-1}\right\|_{\mathrm{cb}}=$ $\left\|\psi^{-1}\right\|_{\text {col-cb }}$, where $\|\psi\|_{\text {col-cb }}:=\sup \left\{\left\|\left(\psi\left(h_{1}\right), \ldots, \psi\left(h_{m}\right)\right)^{t}\right\|\right\}$, where the supremum is extended over all $m \geq 1$ and all column vectors with $\left\|\left(h_{1}, \ldots, h_{m}\right)^{t}\right\| \leq 1$. 
Lemma 3.1. $\|\psi\|_{\text {row-cb }}=\sqrt{k}$ and $\left\|\psi^{-1}\right\|_{\text {col-cb }}=\sqrt{\frac{n}{n-k+1}}$.

Proof. Let $A=\left(C_{h_{1}}^{n, 1}, \ldots, C_{h_{m}}^{n, 1}\right)$ and $B=\left(C_{h_{1}}^{n, k}, \ldots, C_{h_{m}}^{n, k}\right)$. We show first that $\|B\| \leq \sqrt{k}\|A\|$. We have $A A^{*}=\sum_{i=1}^{m} C_{h_{i}}^{n, 1} C_{h_{i}}^{n, 1 *}=\sum_{i=1}^{m} h_{i} \otimes \overline{h_{i}}$ and $B B^{*}=$ $\sum_{i=1}^{m} C_{h_{i}}^{n, k} C_{h_{i}}^{n, k *}$.

Let the (repeated) eigenvalues of $A A^{*}$ be $\lambda_{1} \geq \cdots \geq \lambda_{n}$. By Lemma 2.3(b), we have

$$
\|B\|^{2}=\left\|B B^{*}\right\|=\lambda_{1}+\cdots+\lambda_{k} \leq k \lambda_{1}=k\left\|A A^{*}\right\|=k\|A\|^{2} .
$$

Taking $m=n$ and $h_{j}=e_{j}$, we have $A A^{*}=I$ and $B B^{*}=k I$, proving the first statement of the lemma.

Let $D=A^{t}=\left(C_{h_{1}}^{n, 1}, \ldots, C_{h_{m}}^{n, 1}\right)^{t}$ and $C=B^{t}=\left(C_{h_{1}}^{n, k}, \ldots, C_{h_{m}}^{n, k}\right)^{t}$. We show next that $\|D\| \leq \sqrt{\frac{n}{n-k+1}}\|C\|$. By Lemma 2.3(a), we have $D^{*} D=\sum_{i=1}^{m} C_{h_{i}}^{n, 1 *} C_{h_{i}}^{n, 1}=$ $\sum_{i=1}^{m}\left\|h_{i}\right\|^{2}$; and $C^{*} C=\sum_{i=1}^{m} C_{h_{i}}^{n, k *} C_{h_{i}}^{n, k}$. Since $C^{*} C$ is a square matrix of size $\left(\begin{array}{c}n \\ k-1\end{array}\right)$, again by Lemma 2.3(a),

$$
\left\|C^{*} C\right\|\left(\begin{array}{c}
n \\
k-1
\end{array}\right) \geq \operatorname{tr}\left(C^{*} C\right)=\left(\begin{array}{l}
n-1 \\
k-1
\end{array}\right) \sum_{i=1}^{m}\left\|h_{i}\right\|^{2} .
$$

Therefore,

$$
\frac{\|D\|^{2}}{\|C\|^{2}}=\frac{\sum_{i=1}^{m}\left\|h_{i}\right\|^{2}}{\left\|C^{*} C\right\|} \leq \frac{\sum_{i=1}^{m}\left\|h_{i}\right\|^{2}}{\frac{\left(\begin{array}{c}
n-1 \\
k-1
\end{array}\right)}{\left(\begin{array}{c}
n \\
k-1
\end{array}\right)} \sum_{i=1}^{m}\left\|h_{i}\right\|^{2}}=\frac{n}{n-k+1} .
$$

Taking $m=n$ and $h_{i}=e_{i}$, we have $D^{*} D=n$. On the other hand,

$$
C_{e_{i}}^{n, k *} C_{e_{i}}^{n, k}\left(e_{i_{1}} \wedge \cdots \wedge e_{i_{k-1}}\right)=0
$$

if $i \in\left\{i_{1}, \ldots, i_{k-1}\right\}$ and equal to $e_{i_{1}} \wedge \cdots \wedge e_{i_{k-1}}$ otherwise. Hence $C^{*} C=(n-k+1) I$, proving the second statement.

Theorem 3. $d_{\mathrm{cb}}\left(H_{n}^{k}, H_{n}^{1}\right)=\sqrt{\frac{k n}{n-k+1}}$, for $1 \leq k \leq n$.

Proof. By [12, Theorem 3.1], and the first paragraph of its proof, $\mathrm{d}_{\mathrm{cb}}\left(H_{n}^{k}, H_{n}^{1}\right)=$ $\|\psi\|_{\mathrm{cb}}\left\|\psi^{-1}\right\|_{\mathrm{cb}}$. Now apply Lemma 3.1 and the remarks just preceding it.

Not surprisingly, we obtain the result published first by Mathes ([6, Prop. 7], 9, p. 21]).

Corollary 3.2. $d_{\mathrm{cb}}\left(R_{n}, C_{n}\right)=n$.

Symmetry considerations in Theorem 3 suggest $\mathrm{d}_{\mathrm{cb}}\left(H_{n}^{k}, H_{n}^{n}\right)=\mathrm{d}_{\mathrm{cb}}\left(H_{n}^{n-k+1}, H_{n}^{1}\right)$, which is proved by exactly the same methods. Hence we obtain the following corollary, which is the answer to Problem 1 in [7.

Corollary 3.3. $d_{\mathrm{cb}}\left(H_{n}^{k}, H_{n}^{n}\right)=\sqrt{\frac{(n-k+1) n}{k}}$, for $1 \leq k \leq n$.

Proof. For a fixed $k$, let $\xi: H_{n}^{n} \rightarrow H_{n}^{k}$ be the isometry given by $\xi\left(C_{e_{i}}^{n, n}\right)=C_{e_{i}}^{n, k}$.

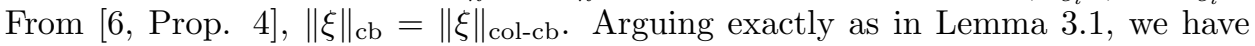
$\|\xi\|_{\text {col-cb }}=\sqrt{n-k+1}$ and $\left\|\xi^{-1}\right\|_{\text {row-cb }}=\sqrt{n / k}$. By [12, Theorem 3.1] and the first paragraph of its proof, $\mathrm{d}_{\mathrm{cb}}\left(H_{n}^{k}, H_{n}^{n}\right)=\|\xi\|_{\mathrm{cb}}\left\|\xi^{-1}\right\|_{\mathrm{cb}}$. 
Remark 3.4. It is curious to note that by Theorem 3 and its two corollaries, $\mathrm{d}_{\mathrm{cb}}\left(H_{n}^{k}, H_{n}^{n}\right) \mathrm{d}_{\mathrm{cb}}\left(H_{n}^{k}, H_{n}^{1}\right)=\mathrm{d}_{\mathrm{cb}}\left(H_{n}^{n}, H_{n}^{1}\right)$, so that in the metric space of all operator spaces of dimension $n$ ([9, page 335]), the three points $H_{n}^{k}, H_{n}^{1}, H_{n}^{n}$ form a degenerate triangle.

Since $H_{n}^{k}$ is distinct from row or column Hilbert space, new ideas will be needed to solve the following problem.

Problem 1. Find $\mathrm{d}_{\mathrm{cb}}\left(H_{n}^{k_{1}}, H_{n}^{k_{2}}\right)$ for $1<k_{1}<k_{2}<n$.

We have already mentioned in the Introduction that $C_{n}^{*}=R_{n}$ and $R_{n}^{*}=C_{n}$ in the category of operator spaces. Hence $H_{n}^{1}$ and $H_{n}^{n}$ are operator space duals of each other. The following problem is therefore of interest, and its solution would certainly lead to insight into Pisier's question on the operator space dual of $\Phi_{n}, 9$, page 175].

Problem 2. Find the operator space dual of $H_{n}^{k}$.

\section{REFERENCES}

1. Jonathan Arazy and Yaakov Friedman, Contractive projections in $C_{1}$ and $C_{\infty}$, Mem. Amer. Math. Soc. 13 (1978), no 200. MR0481219 (82b:47023)

2. David P. Blecher and Christian Le Merdy, Operator algebras and their modules - an operator space approach, Clarendon Press, Oxford, 2004. MR2111973

3. Edward Effros and Zhong-Jin Ruan, Operator Spaces, Oxford University Press, 2000. MR:1793753 (2002a:46082)

4. Yaakov Friedman and Bernard Russo, Solution of the contractive projection problem, J. Funct. Anal. 60 (1985), 56-79. MR.0780104 (87a:46115)

5. Richard V. Kadison and John R. Ringrose, Fundamentals of the Theory of Operator Algebras, Volume II, Academic Press, 1986. MR0859186 (88d:46106)

6. Ben Mathes, Characterizations of row and column Hilbert space, J. London Math. Soc. (2) 50 (1994), 199-208. MR1277763 (96b:47048)

7. Matthew Neal and Bernard Russo, Contractive projections and operator spaces, Trans. Amer. Math. Soc. 355 (2003), 2223-2362. MR.1973989 (2004c:46117)

8. Vern Paulsen, Completely bounded maps and operator algebras, Cambridge Studies in Advanced Mathematics 78, Cambridge University Press, Cambridge, 2002. MR1976867 (2004c:46118)

9. Gilles Pisier, Introduction to Operator Space Theory, Cambridge University Press, 2003. MR.2006539 (2004k:46097)

10. A. Guyan Robertson, Injective matricial Hilbert spaces, Math. Proc. Cambridge Philos. Soc. 110 (1991), 183-190. MR.1104613 (93d:46101)

11. Takeo Yokonuma, Tensor spaces and exterior algebra, Translations of Mathematical Monographs, Volume 108, American Mathematical Society, 1992. MR1187759 (93j:15020)

12. Chun Zhang, Completely bounded Banach-Mazur distance, Proc. Edinburgh Math. Soc. 40 (1997), 247-260. MR 1454020 (98e:46021)

Department of Mathematics and Computer Science, Denison University, Granville, OнIо 43023

E-mail address: nealm@denison.edu

Department of Mathematics, University of California, Irvine, California 92697-3875

E-mail address: brusso@math.uci.edu 